\title{
Manipulation of light in MIM plasmonic waveguide systems
}

\author{
LU Hua ${ }^{\dagger}$, WANG GuoXi ${ }^{\dagger} \&$ LIU XueMing ${ }^{*}$ \\ State Key Laboratory of Transient Optics and Photonics, Xi'an Institute of Optics and Precision Mechanics, Chinese Academy of Sciences, Xi'an \\ 710119, China
}

Received March 20, 2013; accepted May 10, 2013; published online July 22, 2013

\begin{abstract}
Plasmonic waveguides that allow deeply subwavelength confinement of light provide an effective platform for the design of ultracompact photonic devices. As an important plasmonic waveguide, metal-insulator-metal (MIM) structure supports the propagation of light in the nanoscale regime at the visible and near-infrared ranges. Here, we focus on our work in MIM plasmonic waveguide devices for manipulating light, and review some of the recent development of this topic. We introduce MIM plasmonic wavelength filtering and demultiplexing devices, and present the electromagnetic induced transparency (EIT)-like and Fano resonance effects in MIM waveguide systems. The slow-light and rainbow trapping effects are demonstrated theoretically. These results pave a way toward dynamic control of the special and useful optical responses, which actualize some new plasmonic waveguide-integrated devices such as nanoscale filters, demultiplexers, sensors, slow light waveguides, and buffers.
\end{abstract}

surface plasmons, coupled resonators, optical filters, wavelength demultiplexers, slow light, rainbow trapping

Citation: Lu H, Wang G X, Liu X M. Manipulation of light in MIM plasmonic waveguide systems. Chin Sci Bull, 2013, 58: 3607-3616, doi: 10.1007/s11434$013-5989-6$

Surface plasmon polaritons (SPPs) are electromagnetic waves coupled to the oscillations of conduction electrons propagating along the metal-dielectric interface with exponentially decaying field in the direction perpendicular to the interface [1]. SPPs have been considered as one of the most promising energy and information carries to overcome the traditional diffraction limit of light and manipulate light on the subwavelength scale [1-3]. These features open up a pathway toward ultracompact light-guiding structures, which allow the subwavelength confinement of optical modes. The plasmonic waveguides have shown the dramatic potential to guide subwavelength optical modes. So far, a variety of plasmonic waveguiding structures have been proposed, such as metallic wedges [4], grooves [2], nanowires [5], and metal-insulator-metal (MIM) structures [6-10]. MIM waveguides have the deep-subwavelength confinement of light with an acceptable propagation length

$†$ These authors contributed equally to this work.

*Corresponding author (email: liuxueming72@yahoo.com; liuxm@opt.ac.cn) for SPP propagation. The unique features of MIM waveguides can be utilized to realize nanoscale photonic functionality and circuitry [11].

In this review, we mainly focus on our research about the manipulating properties in the MIM plasmonic waveguides. Due to the limit of this small topic, an amount of research in the plasmonic field will be omitted, for example, the important applications of SPPs for the enhancement of nonlinear effects [12], beam focusing [13,14], polarization analyzer [15], optical amplifier [16], and so on. We review some recent development of MIM plasmonic waveguide devices. Especially, plasmonic wavelength filtering and demultiplexing have been introduced. The analogue of electromagnetic induced transparency (EIT) and Fano resonances and their applications are presented in the MIM plasmonic waveguide systems. The slow light and rainbow trapping in the MIM waveguides are demonstrated. These results may provide some useful information for attracting researchers' interests in further investigation of the control of light and its applications in the plasmonic waveguides. 


\section{Wavelength filtering and demultiplexing in MIM plasmonic waveguides}

To realize the wavelength-selective functions in MIM waveguides, some SPP reflectors have been proposed [17-23]. For example, Wang et al. [17] reported SPP Bragg reflectors based on a metal heterowaveguide constructed by alternately stacking two kinds of metal gap waveguides. Low-loss SPP Bragg reflectors were realized through periodically changing the dielectric materials between the MIM waveguide [18,19]. Liu et al. [20] investigated a SPP wide bandgap reflector with relatively wide bandgap realized by modulating the waveguide width and inserting the dielectric materials. We proposed a SPP reflector by means of a quasi-chirped technique in the Bragg-grating-based MIM waveguide. This SPP reflector possesses the broad bandgap and low sidelobes [21]. We also demonstrated a plasmonic Bragg filter with a high-channel-count based on the MIM Fibonacci-sequence gratings [22]. To overcome the complexity of fabrication and decrease the length of plasmonic Bragg reflectors, some simple MIM plasmonic waveguide filters have been proposed, plasmonic filters with stubs [23-26], disk/ring resonators [27-32], and rectangular resonators [33-35]. As key factors in these plasmonic nanostructures, coupled resonators will be principal elements due to their simplicity, symmetry, and ease of fabrication. Wavelength demultiplexers (WDMs), which can filter specific wavelengths in different channels, will play very important role in the all-optical systems [36]. Based on MIM coupled resonators, some WDM structures were proposed [37-43]. Noual et al. [37] reported two-dimensional nanoscale Y-bent plasmonic waveguides with nanocavities for demultiplexing of the telecommunication wavelengths. To further promote the miniaturization, a plasmonic triplewavelength demultiplexer based on nano-capillary resonators (F-P cavities) was investigated by Huang et al. [38]. Recently, an improved compact WDM structure based on arrayed MIM drop cavities was proposed by $\mathrm{Hu}$ et al. [39].
However, the transmission efficiencies of above plasmonic WDMs are too low. In our recent work, a special focus has been put on the schemes to solve this problem [40-43].

Different from the demultiplexing structure in [39], the reflection cavities are introduced in the MIM waveguide. Under the condition that the reflection and drop cavity have the same resonance frequency, the transmission efficiency of each drop filter can be significantly enhanced when the distance between drop and reflection cavities satisfies $D=(2 m+1) \pi /\left(2 n_{\text {eff }} k_{0}\right)(m=0,1,2, \cdots)$. Here, $n_{\text {eff }}$ denotes the effective refractive index of SPP mode, which can be obtained by solving the dispersion equation [43]. $k_{0}=2 \pi / \lambda$ represents the wave vector of incident light in vacuum. For the miniaturization of architectures, $m$ is set as zero. Based on this principle, a highly efficient plasmonic triple-wavelength demultiplexer shown in Figure 1(a) is designed to investigate the transmission response. The geometrical parameters can be seen in [43]. The operating wavelengths of channel drop filters are 928, 820, and $712 \mathrm{~nm}$, respectively. In the bus waveguide, the transmitted powers at these wavelengths are effectively suppressed by reflection cavities. According to the above equation, the optimal distances $D_{1}, D_{2}$, and $D_{3}$ are 166, 145, and $125 \mathrm{~nm}$, respectively. As depicted in Figure 1(b), transmission spectra in the drop waveguides are calculated by the finite-difference time-domain (FDTD) simulations [44]. It is found that the transmission efficiency of each channel is up to 0.7 and improved by more than $50 \%$ when compared to that without reflection cavities.

\section{Electromagnetic induced transparency (EIT)- like and Fano resonance effects in MIM plas- monic waveguides}

Electromagnetically induced transparency (EIT) is a phenomenon that occurs in atomic systems owing to the quantum interference between the excitation pathways to the atomic upper level $[45,46]$. The EIT effect has promising
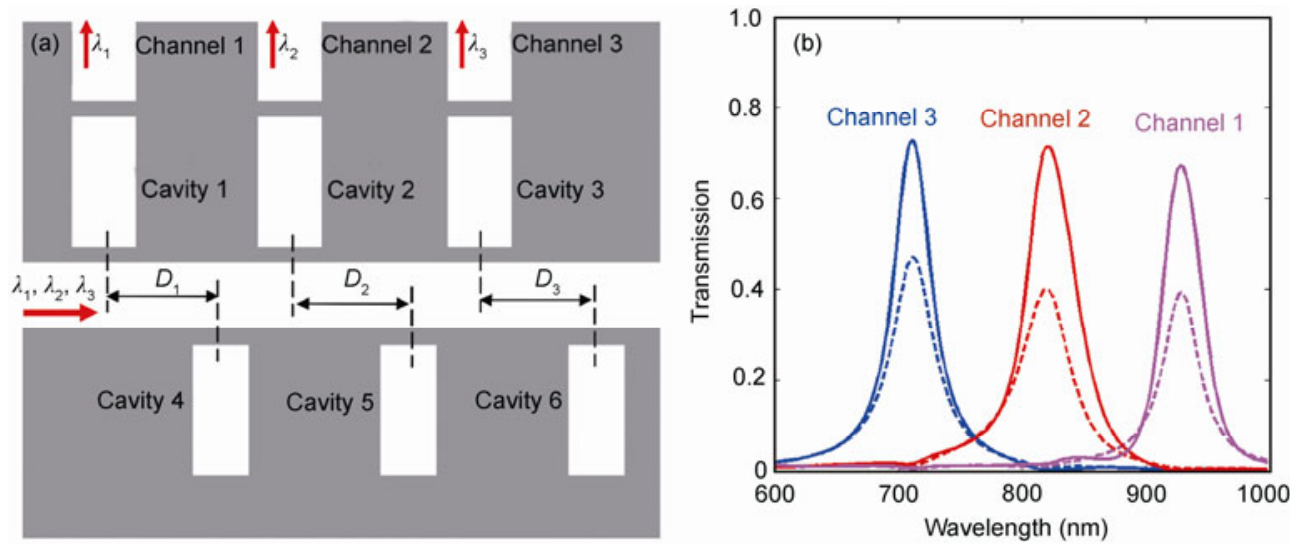

Figure 1 (Color online) (a) Schematic diagram of a plasmonic triple-wavelength demultiplexer; (b) transmission spectra from the drop filters in the plasmonic waveguide without (dashed curves) and with (solid curves) the reflection cavities. From [43], (C) Optical Society of America. 
applications in nonlinear optical process, ultrafast switching, and optical data storage owing to the strong dispersion in the transparency windows $[46,47]$. However, on-chip applications of the atomic EIT are severely limited by the rigorous conditions [48]. The theoretical analysis and experimental observations demonstrate that an optical phenomenon analogous to EIT can occur in the coupled optical resonator systems, which is regarded as coupled-resonator induced transparency (CRIT) [49-53]. The CRIT can be described with the terms used in the EIT physics, where the role of the "atom" is played by the cavity, the "atomic transition" is acted by the cavity mode, and the "driving laser beam" is represented by the strong coupling between the adjacent cavities [54]. Recently, the plasmonic analogue of EIT has been investigated intensively in metamaterials [55-57]. The EIT-like performance in MIM plasmonic waveguide-resonator systems is also significant for the dynamic control and manipulation of light in the nanostructures. Bozhevolnyi et al. [58] and Han et al. [59] described two physical pictures for realizing the EIT-like response. The first (bare-state) picture suggests employing radiative and subradiant resonators that are closely placed and mutually coupled. The second (dressed-state) picture suggests using detuned resonators that are both coupled to a bus waveguide. The latter scheme is essential for the observation of EIT-like and slow-light effects. Han et al. [59] demonstrated the realization of on-chip plasmonic analogue of EIT in integrated plasmonic devices using detuned Fabry-Perot resonators coupled to a MIM waveguide. The MIM structure exhibits the pronounced EIT-like spectrum and group index of $\sim 26$ in near-infrared range. Park et al. [60] realized high-performance plasmonic modulators through controlling the asymmetry of plasmon induced transparency in the MIM waveguide platform. As an important phenomenon, multi-EIT-like spectral response can be realized in multilevel coherently coupled cavities [51]. We also focus on the multi-EIT behavior in a MIM waveguide-resonator system, as shown in Figure 2(a).

Here, we perform triple-resonator-coupled system as an example to investigate the multi-peak EIT-like response. The geometrical parameters can be seen in [61]. As depicted in Figure 2(b), transmission spectrum calculated by the theoretical model is in accordance with the numerical simulation. Dual induced-transparency peaks are found in the areas of strong dispersion generated in our plasmonic system. Transmission phase shift, optical delay line, and group indices are respectively depicted in Figure 2(c)-(e). The group indices and quality factors of transparency resonances with high transmission can reach the level of $\sim 35$ and $\sim 200$, respectively. We also investigated the first picture of EIT-like effect in the MIM waveguides [62]. An optical effect analogous to EIT was observed in nanoscale plasmonic resonator systems. Cui et al. [63] investigated the optical bistability effect with low threshold intensity by using this kind of EIT-like effect in MIM waveguide-coupled

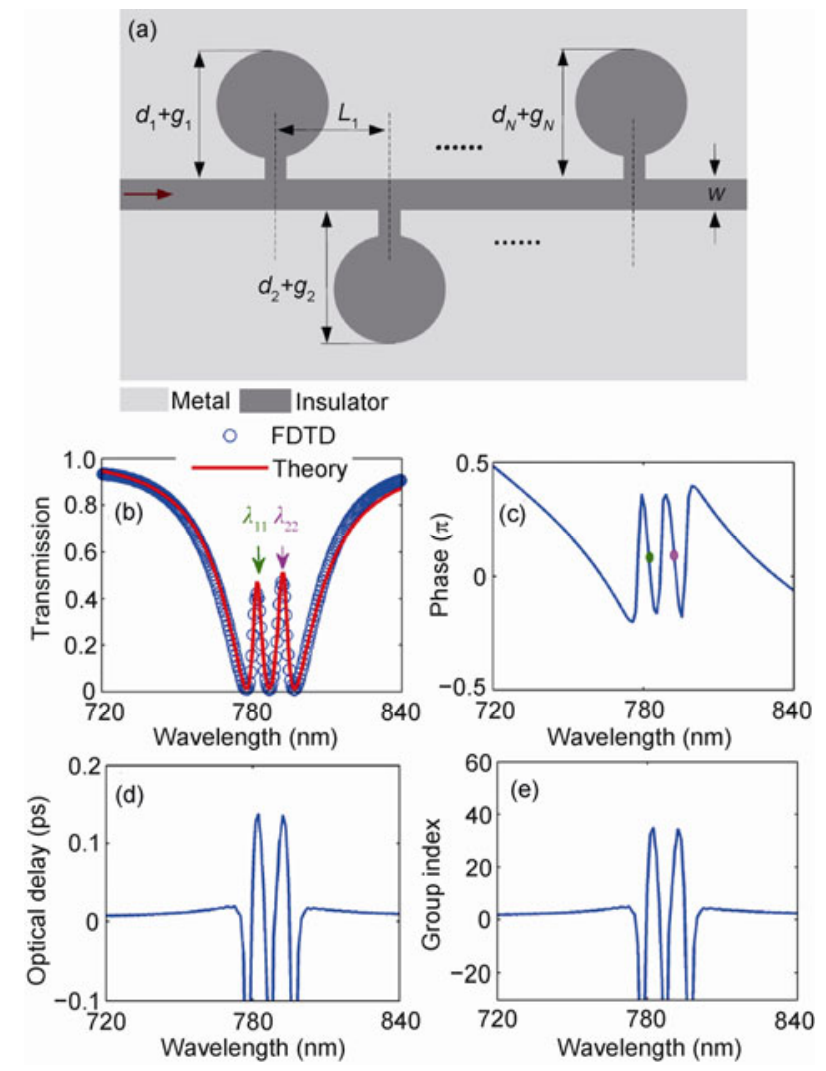

Figure 2 (Color online) (a) Schematic diagram of plasmonic multiresonator waveguide systems; (b) simulation (dotted curve) and theoretical (solid curve) transmission spectra in the triple-resonator-coupled waveguide; (c) corresponding transmission phase shift; (d) optical delay line; (e) group indices. From [61], (c) American Physical Society.

\section{resonator system.}

As depicted in Figure 1(a) of [64], another waveguideresonator configuration exhibits the plasmonic analogue of EIT. It is found that a high transmission peak appears in the background of a broad transmitted dip and possesses a nearly linear red-shift with increasing the length of the stub waveguide, as shown in Figure 3. The plasmonic EIT-like feature enables the effective realization of nanoscale high-

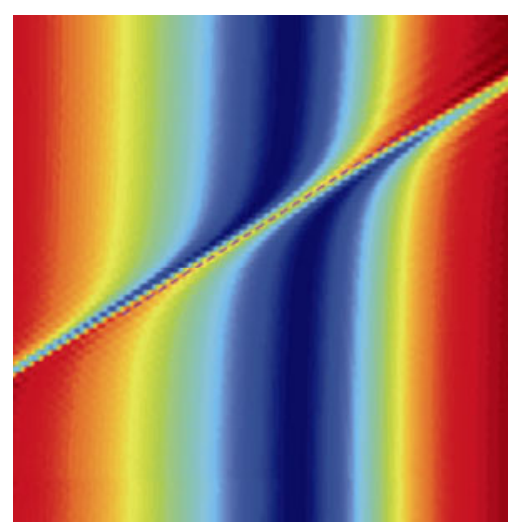

Figure 3 (Color online) Evolution of spectral transmittance with the length of the stub waveguide. From [64], (C) IOP Publishing Ltd. 
channel-count bandpass filters. As shown in Figure 4(a), we propose a plasmonic configuration with cascading fundamental filtering units with adjacent separation $D_{i}(i=1,2, \cdots$ $N-1)$. As an example, four filtering units $(N=4)$ are designed to realize four-channel bandpass plasmonic filters. The geometrical parameters can be seen in [64]. As depicted in Figure 4(b), the EIT-like spectral peaks exhibit nearly identical interval and height. Meanwhile, the forbidden regions between the peaks are sufficiently low. When the third stub waveguide is removed, the corresponding pass band around $850 \mathrm{~nm}$ disappears, as can be seen in Figure 4(c). Figure 4(d) illustrates that the side channels are suppressed in the absence of the first and last stub waveguides. This feature can be employed to broad the sidelobes of the plasmonic filters.

Fano resonance was discovered by Ugo Fano, which originates from the quantum-mechanical interference between a discrete excited state of an atom and a continuum sharing the same energy level [65,66]. Different from the Lorentzian resonance, the Fano resonance possesses distinctly asymmetric line profile [67]. Especially, asymmetric Fano resonances were found in the classical optical systems [67]. Recently, Fano resonances have been observed in plasmonic structures [68-73]. Under certain conditions a Fano resonance can also be essentially regarded as the classical analogue of EIT [55]. These conditions are: (1) sufficiently small frequency detuning between two coupled resonances; (2) strongly contrasting resonance line widths; and (3) appropriate resonance amplitudes [66]. The specific feature of the Fano resonance promises applications in sensors [66]. As shown in Figure 5(a), we have proposed a plasmonic sensor based on Fano resonance in a plasmonic
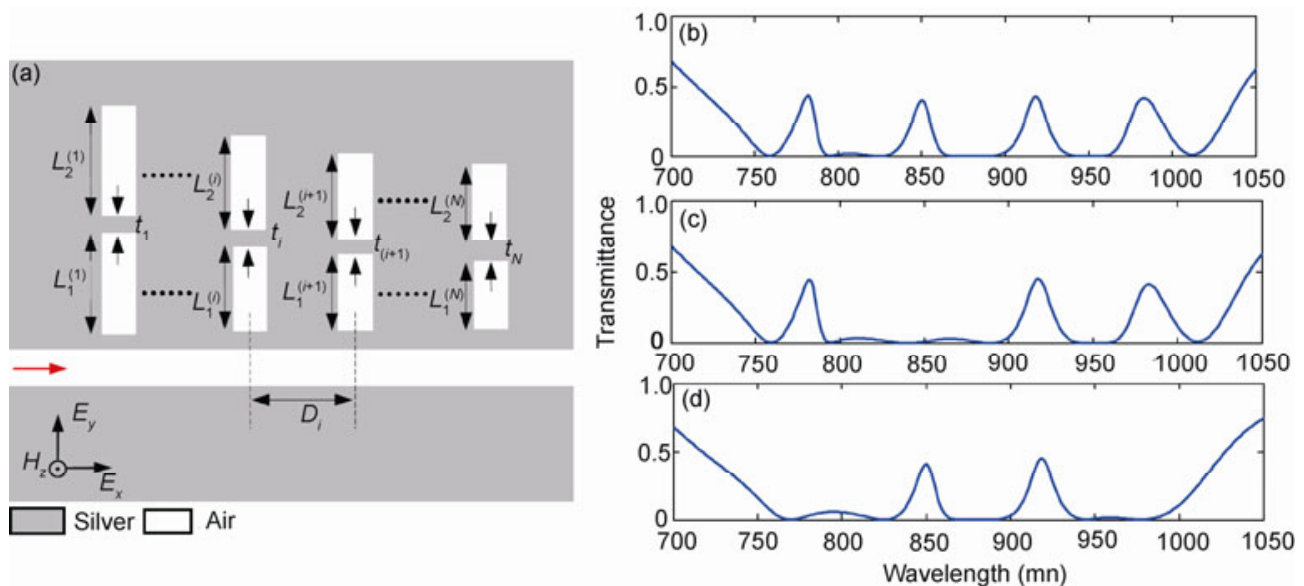

Figure 4 (Color online) (a) Schematic diagram of the plasmonic filtering structure with arrayed coupled cavities and stub waveguides; (b) spectral transmittance of the plasmonic waveguide with four filtering units; (c) spectral transmittance without the third stub waveguide; (d) spectral transmittance without the first and last stub waveguides. From [64], (C IOP Publishing Ltd.

(a)
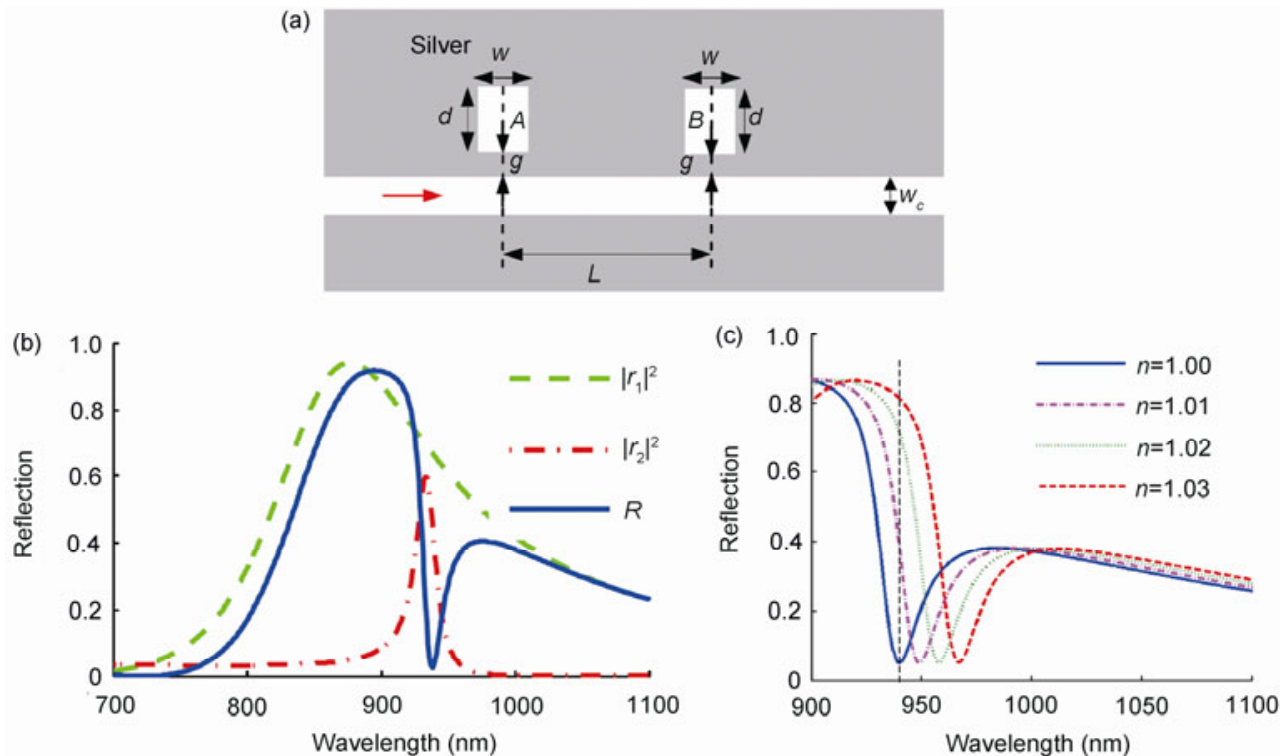

Figure 5 (Color online) (a) Schematic diagram of the MIM plasmonic dual-resonator-coupled waveguide; (b) spectral intensities of reflection amplitudes $r_{1}$ (dotted curve), $r_{2}$ (dash-dotted curve), and $r$ (solid curve); (c) reflection spectra obtained by FDTD simulations for different refractive indices. From [74], (C) Optical Society of America. 
waveguide-resonator system, which consists of a MIM waveguide coupled with a pair of nanoresonators. The geometrical parameters can be seen in [74]. The spectral features of plasmonic waveguide-resonator systems were investigated by the temporal coupled-mode theory [75]. Figure 5(b) shows that the total reflection spectrum $R$ can be regarded as the coherent interference of $r_{1}$ with a broad bandwidth (a continuum level) and $r_{2}$ with narrow bandwidth (a discrete level) [76]. The Fano resonance dip exhibits a sensitive shift with the refractive index change of the dielectric, as seen in Figure 5(c). This feature provides an excellent scheme for the applications toward nanoscale sensing [77]. The sensitivity (nm/RIU) is the shift in the wavelength of the Fano resonance dip per unit change of refractive index $(n)$ [78]. Our plasmonic nanosensor possesses the sensitivity of $\sim 900 \mathrm{~nm} / \mathrm{RIU}$. According to the definition of a figure of merit (FOM) introduced by Becker et al. [79], the maximum value of FOM* is about 500 .

\section{Slow light in MIM plasmonic waveguides}

Stub structures, as important elements in microwave field, can be introduced into the MIM waveguides for the manipulation of light at the nanoscale [24,26,80,81]. Recently, some analytical methods have been reported to account for the optical properties of stub waveguides [82-85]. Especially, the microwave transmission line was proposed to characterize the transmission properties of MIM stub waveguides [7]. Liu et al. [82] developed the transmission line theory for lossless waveguide, which can easily obtain the transmission and dispersion properties. Successively, Pannipitiya et al. [83] proposed an improved transmission model for dissipative MIM waveguides with stub structures. In our work, the improved model is also employed to calculate the transmission and dispersion properties for our MIM stub structures.

To slow down the propagation speed of light, even to coherently trap and store optical pulses, has drawn much attention for its profound applications [86]. Recently, a variety of structures have been reported experimentally and theoretically to realize the slow light effect [84,85,87-90]. Nevertheless, these structures can only be operated at specific resonant wavelength. It is still a challenge to realize slow light over a broad bandwidth. Aiming at this problem, the concept of "rainbow trapping" was proposed by Tsakmakidis et al. [91]. "Rainbow trapping" phenomenon was realized on metal surfaces [92]. MIM waveguides also have dramatic potential for slow-light devices [93-96].

Here, we introduce the slow-light effect in a MIM plasmonic waveguide with quasi-period stub structures, as shown in Figure 6(a). The geometrical parameters can be seen in [96]. Figure 6(b) shows the evolution of propagation constant at different grating depths. The results reveal that the cutoff frequency has a red-shift with the increase of the grating depth. Figure 6(c) depicts the group index $c / v_{\mathrm{g}}$ as a function of the incident frequency at a given grating depth. It is found that the group velocity $v_{\mathrm{g}}(\equiv \partial \omega / \partial \beta)$ can be

(a)
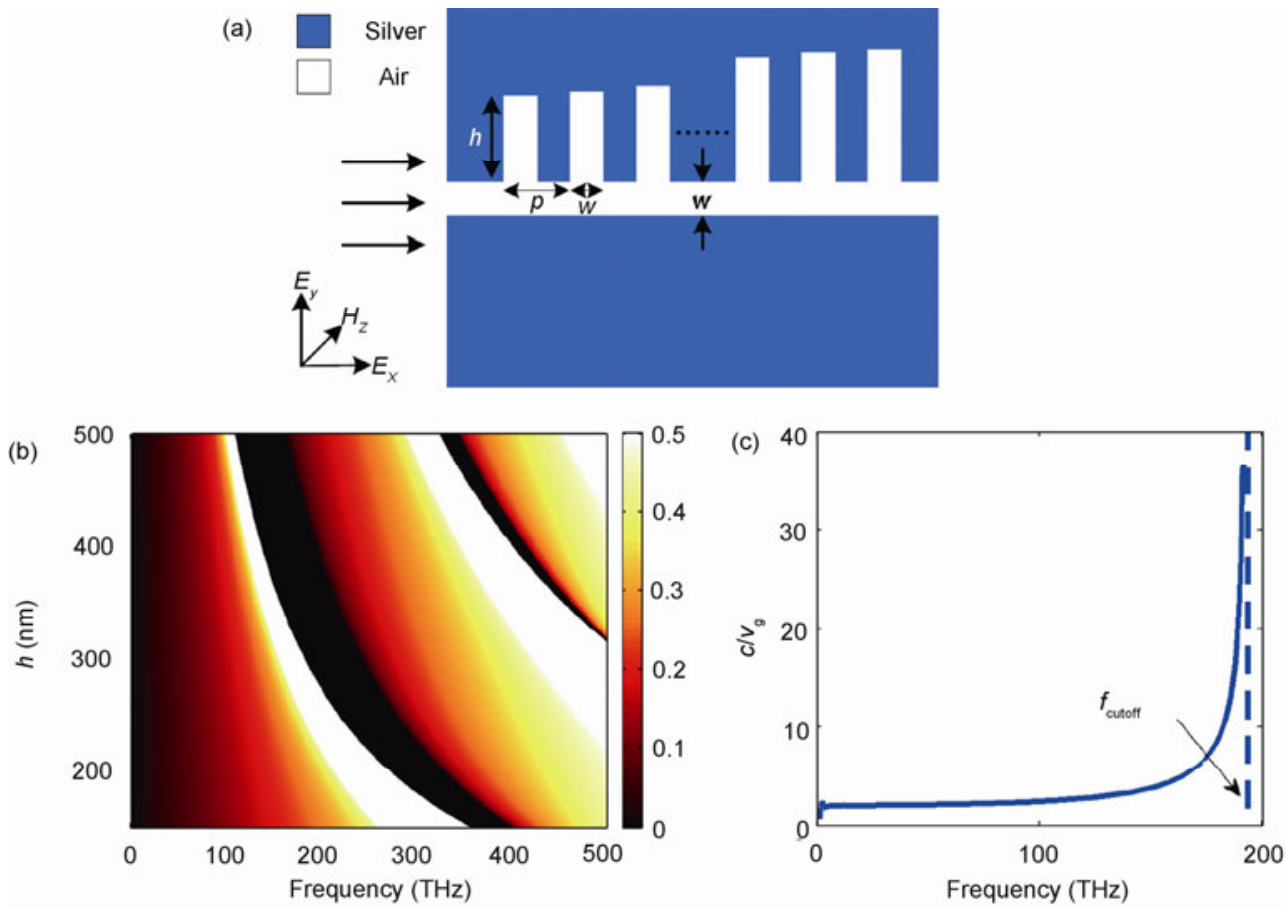

Figure 6 (Color online) (a) Schematic diagram of the plasmonic waveguide with quasi-period stub structures; (b) evolution of propagation constant at different frequencies with the grating depth for $w=50 \mathrm{~nm}$ and $p=200 \mathrm{~nm}$; (c) group index of SPP wave as a function of frequency for the grating depth of 320 $\mathrm{nm}$. The cutoff frequency is about 193.5 THz. From [96], (C) American Institute of Physics. 
slowed down significantly when the incident frequency approaches the cutoff frequency. The quasi-period-stub structure is introduced to enlarge the slow light frequency range. Due to the small graded grating depth, the dispersion relations are supposed to change gradually along the waveguide with the ascending grating depth. Thus, the group velocity of incident light with different frequencies can be greatly reduced at different locations.

We also calculate the dependence of SPP intensity in the stubs on the propagation time and distance. As shown in Figure 7(a), the SPP wave propagates along the $x$ direction with an increasingly slower group velocity, and the strongest intensity corresponds to the localized position of SPPs. The field distributions in Figure 7(b) and (c) show that our plasmonic structure can trap the specific SPP wave at a certain location. It should be noted that, however, the SPP wave can never be completely trapped due to the metallic absorption. To realize the real trap of SPPs, one can incorporate a gain media in the structure to compensate for the metal loss. Thus, the group velocity $v_{\mathrm{g}}$ of SPP wave is expected to be further slowed down or even to be zero.

Generally, some approaches are used to generate slow light, such as EIT effects [97,98], special photonic structures [92], and stimulated Brillouin scattering [99]. Our above study show how to realize slow light effect and "rainbow trapping" in a plasmonic waveguide system. Utilizing the photonic bandgap (PBG) of the proposed structure, slow light can be easily observed close to the PBG bandage [100]. However, the dispersion relation around the operating slow-light point shows a near parabolic property, which denotes that large group velocity dispersion (GVD) parameter may result in the pulse distortion. This feature confines the practical applications of the periodic structures. Moreover, the normalized delay-bandwidth product (NDBP) is not considered, which is regarded as a good candidate to characterize the compromise between the light slowing down factor and the bandwidth [89,101]. The EIT-like effect has attracted much attention for the strong dispersion and slow light propagation in the transparency window [85].

As shown in Figure 8(a), we propose a MIM plasmonic slow-light waveguide based on an analogue of EIT. The geometrical parameters can be seen in [87]. This plasmonic waveguide system can realize a significant slow light with zero GVD parameter, which indicates that the incident pulse can be slowed down without distortion. Meanwhile, the NDBP of the waveguide is as high as 0.522 , implying a high slow-light buffering capacity. As depicted in Figure 8(b), the transmission properties of the MIM waveguide coupled with two stubs are investigated by the improved transmission model. By appropriately tuning the distance between the two stubs, the transmission spectrum exhibits two transmitted dips $\left(f_{1}\right.$ and $\left.f_{3}\right)$ and a transparency peak $\left(f_{2}\right)$. The appearance of the transmission peak is attributed to the destructive interference between the electromagnetic fields from the two stubs. As shown in Figure 8(c), the numerical simulations agree well with the theoretical results.

As shown in Figure 9(a), the dispersion curve is flat and has slightly fluctuation in the frequency range of EIT window, which indicates that a nearly constant group velocity can be achieved. The obtained group index near the peak frequency possesses considerable stability and nearly constant value. This indicates that slow light with zero GVD parameter can be obtained in the proposed structure. Thus, an incident optical pulse at the peak frequency will suffer almost no distortion. To validate the analytical results, Figure 9(b) shows the time evolution of the pulse propagating through the plasmonic waveguide system. It is found that the relative pulse shape distortion is only $2.12 \%$ due to the low GVD parameters. The corresponding group index is 21.57, which agrees well with the transmission line results $\left(n_{\mathrm{g}}=22\right)$. In this plasmonic structure, we obtain an excellent buffering capacity with a flat bandwidth over $8.6 \mathrm{THz}$ and a high NDBP value of 0.522 .

In above MIM slow-light waveguides, the influence of metal loss on the trapping performance is not considered. When an arbitrarily small loss is introduced, the slope of the dispersion curve at the PBG edge is not close to zero and SPP wave cannot be stopped completely [102]. It is important to investigate the impact of metal loss on the rainbow trapping performance and explore new methods to
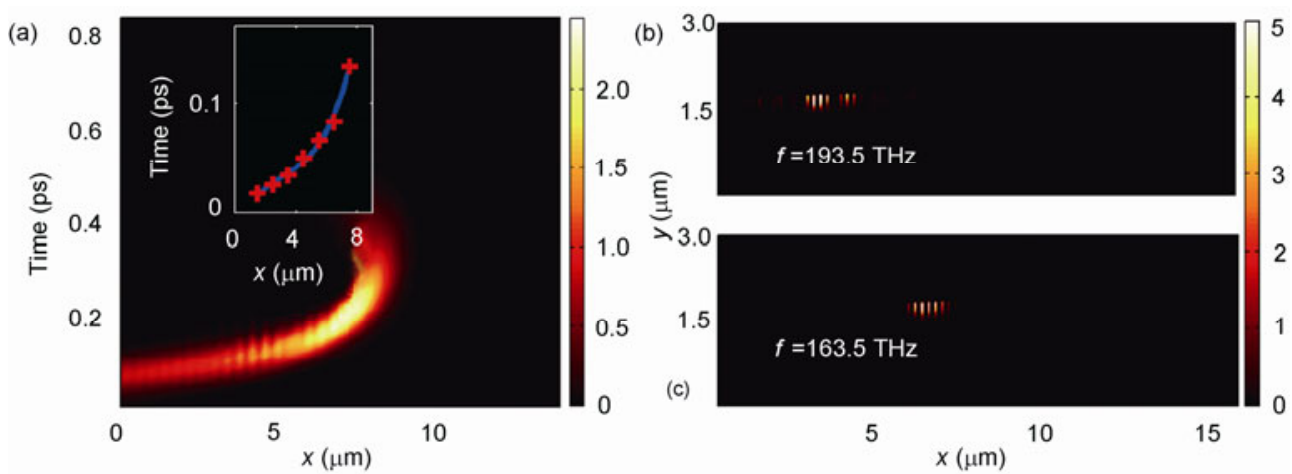

Figure 7 (Color online) (a) Time-dependent field intensity in the stubs. Inset: Arrival time of SPP wave at different spatial positions. (b), (c) Field distributions with the incident frequencies of 193.5 and 163.5 THz. From [96], (C) American Institute of Physics. 

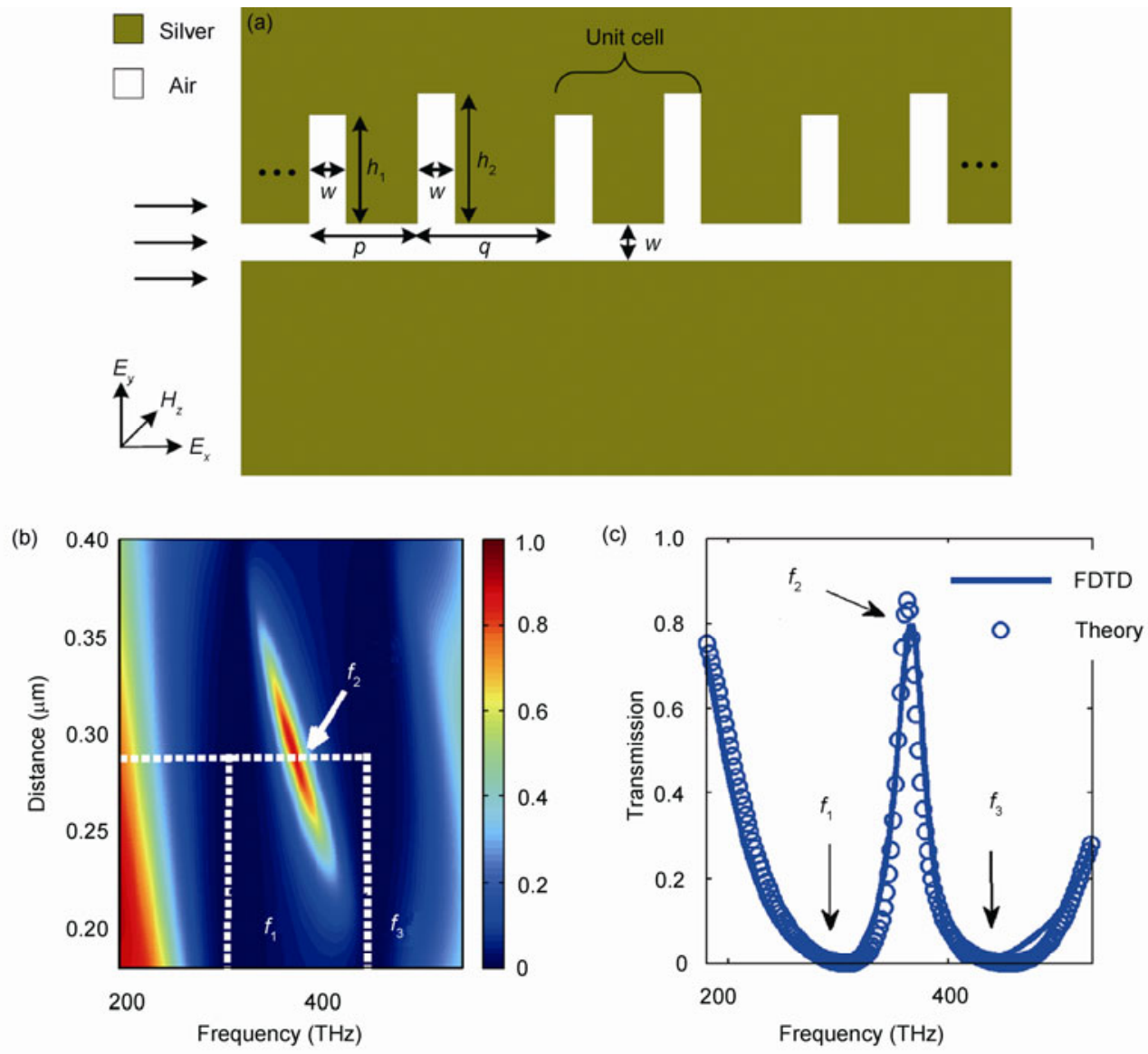

Figure 8 (Color online) (a) Schematic diagram of the plasmonic slow-light waveguide; (b) evolution of transmission spectrum with the distance between the two stubs in a unit cell; (c) transmission spectra from the transmission line theory and numerical simulation for a unit cell. From [87], (C) Optical Society of America.
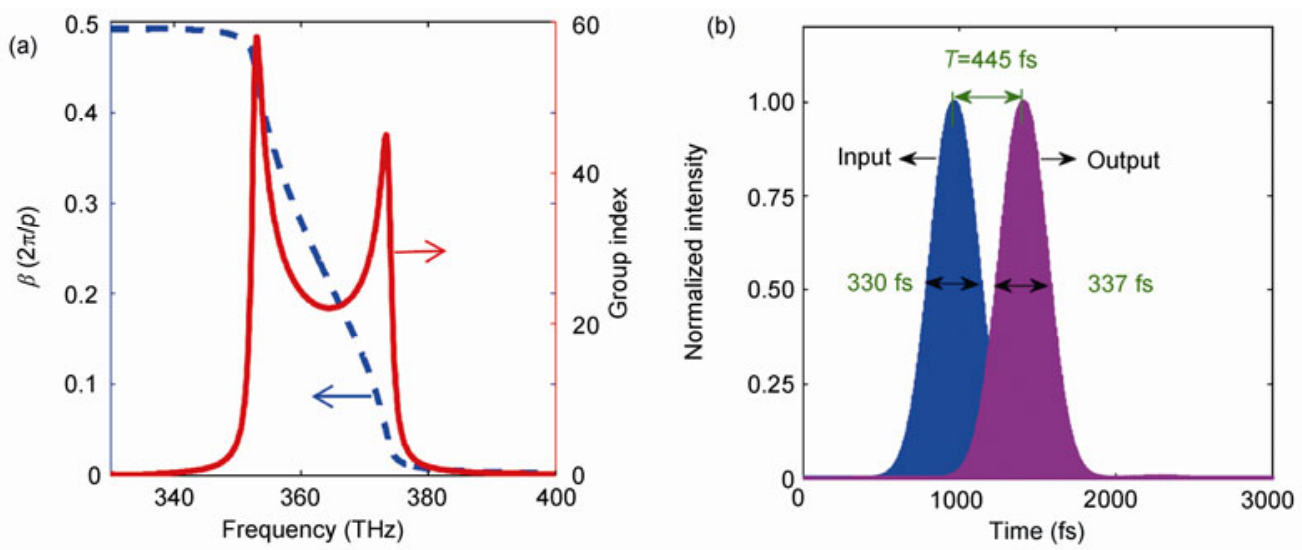

Figure 9 (Color online) (a) Dispersion curves and group index of SPP wave as a function of the frequency; (b) time evolution of normalized intensity profile of SPP pulse propagating through the waveguide system with 11 unit cells. From [87], (C) Optical Society of America.

compensate the metal loss.

Recently, researchers confirmed that MIM tapered waveguides are best suited for slowing and trapping light $[103,104]$. A focus has been put on the tapered plasmonic waveguide, which consists of a gain core layer and metal cladding, as shown in Figure 10(a). The geometrical parameters can be seen in [105]. By incorporating appropriate gain into the waveguide to compensate the metal loss, the zero group velocity is obtained and SPP waves could be trapped in such a tapered MIM waveguide. The detailed analysis can be seen in [106]. As shown in Figure 10(b), when the loss is considered, the forward and backward modes diverge and split into two different parts, the group velocity for either forward or backward no longer declines 


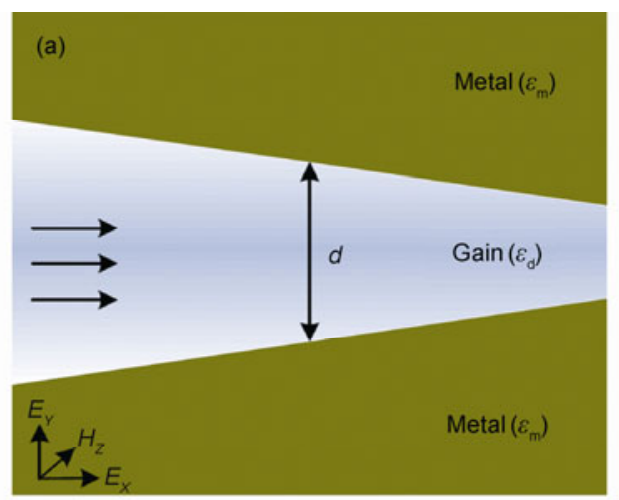

(b)

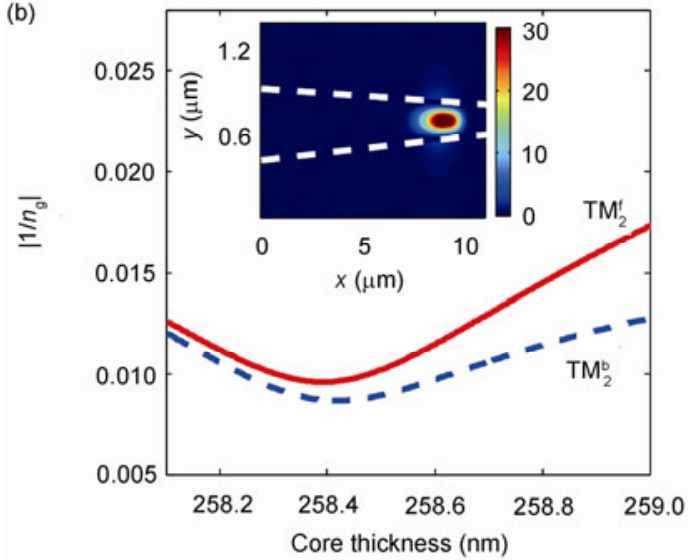

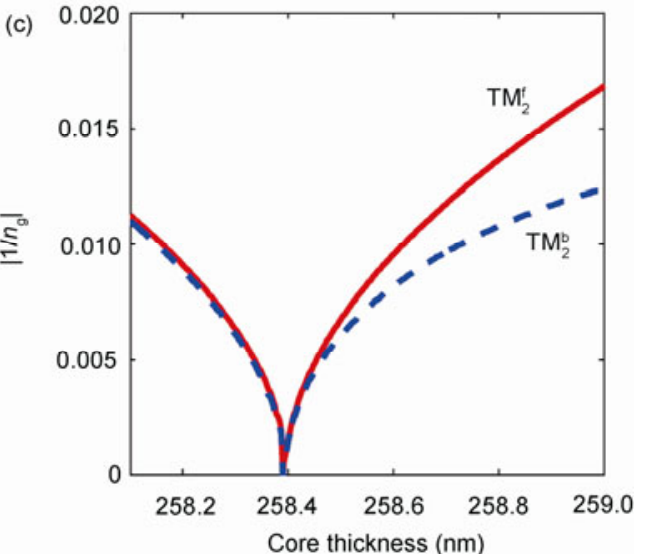

Figure 10 (Color online) (a) Schematic diagram of the tapered plasmonic waveguide. (b) Group index for lossy case. The red and blue curves correspond to the forward and the backward modes. For both the lossless and lossy cases, the core layer has $\varepsilon_{\mathrm{d}}=12.25$. The wavelength of the incident light is $1550 \mathrm{~nm}$. The inset shows the field distribution of a Gaussian pulse with a $100 \mathrm{fs}$ width and $1550 \mathrm{~nm}$ central wavelength propagating through the plasmonic waveguide tapered from 500 to $200 \mathrm{~nm}$ in an $11 \mu \mathrm{m}$ region. (c) Group index with incorporating gain into the core layer. The gain coefficient is about $197 \mathrm{~cm}^{-1}$. From [105], (C) Optical Society of America.

to zero. The metal loss can prevent the group velocity from approaching zero. When the core thickness increases, even though, the forward and backward modes can still keep a relative small group velocity $(<0.01 c)$ near the critical thickness. In the inset of Figure 10(b), the tapered plasmonic waveguide is illuminated by a Gaussian pulse. Simulation results show that a strong field accumulation exhibits at the critical thickness of the waveguide, which indicates a significant slow light effect. When the core thickness is larger than the critical thickness, the plasmonic waveguide only supports the decay mode and SPP wave cannot propagate further. By employing gain material such as semiconductors, the loss effect can be perfectly compensated [106]. As shown in Figure 10(c), the gain compensates the metal loss and forces the forward and backward modes to combine together. At the critical thickness, the group velocity equals zero.

\section{Conclusions}

In this review, we mainly focus on our work about the manipulation of light in the MIM plasmonic waveguides. Es- pecially, plasmonic wavelength filtering and demultiplexing have been introduced. Additionally, the EIT-like and Fano resonance effects in the MIM plasmonic systems have been described. Finally, the slow light and rainbow trapping effects in the MIM waveguides with metal loss and gain compensation have been demonstrated. The optical responses can find applications for plasmonic waveguide devices such as nanoscale filters, demultiplexers, sensors, slow light waveguides, and buffers. Our results and physical analysis would boost the further development of plasmonic waveguides for the excellent control of light in the plasmonic nanostructures.

This work was supported by the National Natural Science Foundation of China (10874239, 10604066 and 11204368).

1 Barnes W L, Dereux A, Ebbesen T W. Surface plasmon subwavelength optics. Nature, 2003, 424: 824-830

2 Bozhevolnyi S I, Volkov V S, Devaux E, et al. Channel plasmon subwavelength waveguide components including interferometers and ring resonators. Nature, 2006, 440: 508-511

3 Gramotnev D, Bozhevolnyi S I. Plasmonics beyond the diffraction limit. Nat Photon, 2010, 4: 83-91

4 Moreno E, Rodrigo S G, Bozhevolnyi S I, et al. Guiding and focusing 
of electromagnetic fields with wedge plasmon polaritons. Phys Rev Lett, 2008, 100: 023901

5 Fang Y, Li Z, Huang Y, et al. Branched silver nanowires as controllable plasmon routers. Nano Lett, 2010, 10: 1950-1954

6 Wang B, Wang G P. Surface plasmon polariton propagation in nanoscale metal gap waveguides. Opt Lett, 2004, 29: 1992-1994

7 Veronis G, Fan S H. Bends and splitters in metal-dielectric-metal subwavelength plasmonic waveguides. Appl Phys Lett, 2005, 87: 131102

8 Gao H, Shi H, Wang C, et al. Surface plasmon polariton propagation and combination in Y-shaped metallic channels. Opt Express, 2005, 13: $10795-10800$

9 Lu H, Liu X M, Wang L, et al. Ultrafast all-optical switching in nanoplasmonic waveguide with Kerr nonlinear resonator. Opt Express, 2011, 19: 2910-2915

$10 \mathrm{Fu} \mathrm{Y,} \mathrm{Hu} \mathrm{X,} \mathrm{Lu} \mathrm{C,} \mathrm{et} \mathrm{al.} \mathrm{All-optical} \mathrm{logic} \mathrm{gates} \mathrm{based} \mathrm{on} \mathrm{nanoscale}$ plasmonic slot waveguides. Nano Lett, 2012, 12: 5784-5790

11 Zia R, Schuller J A, Chandran A, et al. Plasmonics: The next chipscale technology. Mater Today, 2006, 9: 20-27

12 Wurtz G A, Pollard R, Zayats A V. Optical bistability in nonlinear surface-plasmon polaritonic crystals. Phys Rev Lett, 2006, 97: 057402

13 Yin L L, Vlasko-Vlasov V K, Pearson J, et al. Subwavelength focusing and guiding of surface plasmons. Nano Lett, 2005, 5: 1399-1402

14 Steele J M, Liu Z, Wang Y, et al. Resonant and non-resonant generation and focusing of surface plasmons with circular gratings. Opt Express, 2006, 14: 5664-5670

15 Yang S Y, Chen W B, Nelson R L, et al. Miniature circular polarization analyzer with spiral plasmonic lens. Opt Lett, 2009, 34: 3047-3049

16 Leon I D, Berini P. Amplification of long-range surface plasmons by a dipolar gain medium. Nat Photon, 2010, 10: 1-6

17 Wang B, Wang G P. Plasmon Bragg reflectors and nanocavities on flat metallic surfaces. Appl Phys Lett, 2005, 87: 013107

18 Hosseini A, Massoud Y. A low-loss metal-insulator-metal plasmonic Bragg reflector. Opt Express, 2006, 14: 11318-11323

19 Hosseini A, Nejati H, Massoud Y. Modeling and design methodology for metal-insulator-metal plasmonic Bragg reflectors. Opt Express, 2008, 16: 1475-1480

20 Liu J Q, Wang L L, He M D, et al. A wide bandgap plasmonic Bragg reflector. Opt Express, 2008, 16: 4888-4894

21 Gong Y K, Wang L R, Hu X H, et al. Broad-bandgap and low-sidelobe surface plasmon polariton reflector with Bragg-grating-based MIM waveguide. Opt Express, 2009, 17: 13727-13736

22 Gong Y K, Liu X M, Wang L R. High-channel-count plasmonic filter with the metal-insulator-metal Fibonacci-sequence gratings. Opt Lett, 2010, 35: 285-287

23 Park J, Kim H, Lee B. High order plasmonic Bragg reflection in the metal-insulator-metal waveguide Bragg grating. Opt Express, 2008, 16: $413-425$

24 Lin X S, Huang X G. Tooth-shaped plasmonic waveguide filters with nanometeric sizes. Opt Lett, 2008, 33: 2874-2876

25 Gong Y K, Liu X M, Wang L R, et al. Unidirectional manipulation of surface plasmon polariton by dual-nanocavity in a T-shaped waveguide. Opt Commun, 2011, 284: 795-798

26 Tao J, Huang X G, Lin X S, et al. A narrow-band subwavelength plasmonic waveguide filter with asymmetrical multiple-teeth-shaped structure. Opt Express, 2009, 17: 13989-13994

27 Lu H, Liu X M, Mao D, et al. Tunable band-pass plasmonic waveguide filters with nanodisk resonators. Opt Express, 2010, 18: $17922-17927$

28 Lu H, Liu X M, Gong Y K, et al. Multi-channel plasmonic waveguide filters with disk-shaped nanocavities. Opt Commun, 2011, 284 : 2613-2616

29 Wang G X, Lu H. Unidirectional excitation of surface plasmon polaritons in T-shaped waveguide with nanodisk resonator. Opt Commun, 2012, 285: 4190-4193

30 Hosseini A, Massoud Y. Nanoscale surface plasmon based resonator using rectangular geometry. Appl Phys Lett, 2007, 90: 181102

31 Wang T B, Wen X W, Yin C P, et al. The transmission characteristics of surface plasmon polaritons in ring resonator. Opt Express, 2009, 17: $24096-24101$
32 Han Z, Van V, Herman W N, et al. Aperture-coupled MIM plasmonic ring resonators with sub-diffraction modal volumes. Opt Express, 2009, 17: 12678-12684

33 Xiao S, Liu L, Qiu M. Resonator channel drop filters in a plasmon-polaritons metal. Opt Express, 2932, 14: 2932-2937

34 Noual A, Pennec Y, Akjouj A, et al. Nanoscale plasmon waveguide including cavity resonator. J Phys: Condens Matter, 2009, 21: 75301

35 Zhang Q, Huang X G, Lin X S, et al. A subwavelength coupler-type MIM optical filter. Opt Express, 2009, 17: 7549-7554

36 Drezet A, Koller D, Hohenau A, et al. Plasmonic crystal demultiplexer and multiports. Nano Lett, 2007, 7: 1697-1700

37 Noual A, Akjouj A, Pennec Y, et al. Modeling of two-dimensional nanoscale Y-bent plasmonic waveguides with cavities for demultiplexing of the telecommunication wavelengths. New J Phys, 2009, 11: 103020

38 Tao J, Huang X G, Zhu J H. A wavelength demultiplexing structure based on metal-dielectric-metal plasmonic nano-capillary resonators. Opt Express, 2010, 18: 11111-11116

39 Hu F, Yi H, Zhou Z. Wavelength demultiplexing structure based on arrayed plasmonic slot cavities. Opt Lett, 2011, 36: 1500-1502

40 Lu H, Liu X M, Wang L R, et al. Nanoplasmonic triple-wavelength demultiplexers in two-dimensional metallic waveguides. Appl Phys B, 2011, 103: 877-881

41 Wang G X, Lu H, Liu X M, et al. Tunable multi-channel wavelength demultiplexer based on MIM plasmonic nanodisk resonators at telecommunication regime. Opt Express, 2011, 19: 3513-3518

$42 \mathrm{Lu} \mathrm{H}$, Liu X M, Mao D, et al. Analysis of nanoplasmonic wavelength demultiplexing based on MIM waveguides. J Opt Soc Am B, 2011, 28: $1616-1621$

$43 \mathrm{Lu} \mathrm{H}$, Liu X M, Gong Y K, et al. Enhancement of transmission efficiency of nanoplasmonic wavelength demultiplexer based on channel drop filters and reflection nanocavities. Opt Express, 2011, 19: $12885-12890$

44 Taflove A, Hagness S C. Computational Electrodynamics: The Finite-Difference Time-Domain Method. Boston: Artech House, 2005

45 Harris S. Electromagnetically induced transparency. Phys Today, 1997, 50: 36-42

46 Fleischhauer M, Imamoglu A, MarangosRev J P. Electromagnetically induced transparency: Optics in coherent media. Rev Mod Phys, 2005, 77: 633-673

47 Boyd R W, Gauthier D J. Transparency on an optical chip. Nature, 2006, 441: 701-702

48 Kekatpure R D, Barnard E S, Cai W, et al. Phase-coupled plasmon-induced transparency. Phys Rev Lett, 2010, 104: 243902

49 Smith D D, Chang H, Fuller K A, et al. Coupled-resonator-induced transparency. Phys Rev A, 2004, 69: 063804

$50 \mathrm{Xu}$ Q, Sandhu S, Povinelli M, et al. Experimental realization of an on-chip all-optical analogue to electromagnetically induced transparency. Phys Rev Lett, 2006, 96: 123901

51 Yang X, Yu M, Kwong D L, et al. All-optical analog to electromagnetically induced transparency in multiple coupled photonic crystal cavities. Phys Rev Lett, 2009, 102: 173902

52 Naweed A, Farca G, Shopova S I, et al. Induced transparency and absorption in coupled whispering-gallery microresonators. Phys Rev A, 2005, 71: 043804

53 Totsuka K, Kobayashi N, Tomita M. Slow light in coupled-resonatorinduced transparency. Phys Rev Lett, 2007, 98: 213904

54 Xiao Y F, Zou X B, Jiang W, et al. Analog to multiple electromagnetically induced transparency in all-optical drop-filter systems. Phys Rev A, 2007, 75: 063833

55 Zhang S, Genov D A, Wang Y, et al. Plasmon-induced transparency in metamaterials. Phys Rev Lett, 2008, 101: 047401

56 Liu N, Langguth L, Weiss T. Plasmonic analogue of electromagnetically induced transparency at the Drude damping limit. Nat Mater, 2009, 8: 758-762

57 Liu N, Weiss T, Mesch M, et al. Planar metamaterial analogue of electromagnetically induced transparency for plasmonic sensing. Nano Lett, 2010, 10: 1103-1107

58 Bozhevolnyi S I, Evlyukhin A B, Pors A, et al. Optical transparency by detuned electrical dipoles. New J Phys, 2011, 13: 023034 
59 Han Z H, Bozhevolnyi S I. Plasmon-induced transparency with detuned ultracompact Fabry-Perot resonators in integrated plasmonic devices. Opt Express, 2011, 19: 3251-3257

60 Piao X, Yu S, Park N. Control of Fano asymmetry in plasmon induced transparency and its application to plasmonic waveguide modulator. Opt Express, 2012, 20: 18994-18999

61 Lu H, Liu X M, Mao D. Plasmonic analog of electromagnetically induced transparency in multi-nanoresonator-coupled waveguide systems. Phys Rev A, 2012, 85: 053803

$62 \mathrm{Lu} \mathrm{H}$, Liu X M, Mao D, et al. Induced transparency in nanoscale plasmonic resonator systems. Opt Lett, 2011, 36: 3233-3235

63 Cui Y D, Zeng C. Optical bistability based on an analog of electromagnetically induced transparency in plasmonic waveguide-coupled resonators. Appl Opt, 2012, 51: 7482-7486

64 Lu H, Liu X M, Wang G X, et al. Tunable high-channel-count bandpass plasmonic filters based on an analogue of electromagnetically induced transparency. Nanotechnology, 2012, 23: 444003

65 Fano U. Effects of configuration interaction on intensities and phase shifts. Phys Rev, 1961, 124: 1866-1878

66 Luk'yanchuk B, Zheludev N I, Maier S A, et al. The Fano resonance in plasmonic nanostructures and metamaterials. Nat Mater, 2010, 9: 707-715

67 Miroshnichenko A E, Flach S, Kivshar Y S. Fano resonances in nanoscale structures. Rev Mod Phys, 2010, 82: 2257-2298

68 Sarrazin M, Vigneron J P. Bounded modes to the rescue of optical transmission. Europhys News, 2007, 38: 27-31

69 Verellen N, Sonnefraud Y, Sobhani H, et al. Fano resonances in individual coherent plasmonic nanocavities. Nano Lett, 2009, 9: 16631667

70 Fedotov V A, Papasimakis N, Plum E, et al. Spectral collapse in ensembles of metamolecules. Phys Rev Lett, 2010, 104: 223901

71 Rahmani M, Lei D Y, Giannini V, et al. Subgroup decomposition of plasmonic resonances in hybrid oligomers: Modeling the resonance lineshape. Nano Lett, 2012, 12: 2101-2106

72 Lassiter J B, Sobhani H, Knight M W, et al. Designing and deconstructing the Fano lineshape in plasmonic nanoclusters. Nano Lett, 2012, 12: 1058-1062

73 Rahmani M, Luk'yanchuk B, Hong M. Fano resonance in novel plasmonic nanostructures. Laser Photon Rev, 2013, 7: 329-349

74 Lu H, Liu X M, Mao D, et al. Plasmonic nanosensor based on Fano resonance in waveguide-coupled resonators. Opt Lett, 2012, 37: 3780-3782

75 Haus H A. Waves and Fields in Optoelectronics. Englewood Cliffs, NJ: Prentice-Hall, 1984

76 Xiao Y F, Li M, Liu Y C, et al. Asymmetric Fano resonance analysis in indirectly coupled microresonators. Phys Rev A, 2010, 82: 065804

77 Liu N, Weiss T, Mesch M, et al. Planar metamaterial analogue of electromagnetically induced transparency for plasmonic sensing. Nano Lett, 2010, 10: 1103-1107

78 Liu N, Mesch M, Weiss T, et al. Infrared perfect absorber and its application as plasmonic sensor. Nano Lett, 2010, 10: 2342-2348

79 Becker J, Truegler A, Jakab A, et al. The optimal aspect ratio of gold nanorods for plasmonic bio-sensing. Plasmonics, 2010, 5: 161-167

80 Matsuzaki Y, Okamoto T, Haraguchi M, et al. Characteristics of gap plasmon waveguide with stub structures. Opt Express, 2008, 16: $16314-16325$

81 Wang G X, Lu H, Liu X M, et al. Numerical investigation of an all-optical switch in a graded nonlinear plasmonic grating. Nanotechnology, 2012, 23: 444009

82 Liu J L, Fang G Y, Zhao H F, et al. Surface plasmon reflector based on serial stub structure. Opt Express, 2009, 17: 20134-20139

83 Pannipitiya A, Rukhlenko I D, Premaratne M, et al. Improved trans- mission model for metal-dielectric-metal plasmonic waveguides with stub structure. Opt Express, 2010, 18: 6191-6204

84 Yang L, Min C J, Veronis G. Guided subwavelength slow-light mode supported by a plasmonic waveguide system. Opt Lett, 2010, 34: 4184-4186

85 Huang Y, Min C J, Veronis G. Subwavelength slow-light waveguide based on a plasmonic analogue of electromagnetically induced transparency. Appl Phys Lett, 2011, 99: 143117

86 Yanik M F, Fan S H. Stopping light all optically. Phys Rev Lett, 2004, 92: 083901

87 Wang G X, Lu H, Liu X M. Dispersionless slow light in MIM waveguide based on a plasmonic analogue of electromagnetically induced transparency. Opt Express, 2012, 20: 20902-20907

88 Ruan Z C, Qiu M. Slow electromagnetic wave guided in subwavelength region along one-dimensional periodically structured metal surface. Appl Phys Lett, 2007, 90: 201906

89 Baba T. Slow light in photonic crystals. Nat Photon, 2008, 2: 465473

90 Wang G X. Slow light engineering in periodic-stub-assisted plasmonic waveguide. Appl Opt, 2013, 52: 1799-1804

91 Tsakmakidis K L, Boardman A D, Hess O. 'Trapped rainbow' storage of light in metamaterials. Nature, 2007, 450: 397-401

92 Gan Q Q, Fu Z, Ding Y J, et al. Ultrawide-bandwidth slow-light system based on THz plasmonic graded metallic grating structures. Phys Rev Lett, 2008, 100: 256803

93 Wang G X, Lu H, Liu X M. Optical bistability in metal-insulatormetal plasmonic waveguide with nanodisk resonator containing Kerr nonlinear medium. Appl Opt, 2011, 50: 5287-5290

94 Zeng C, Cui Y D. Rainbow trapping of surface plasmon polaritons waves in metal-insulator-metal graded grating waveguide. Opt Commun, 2013, 290: 188-191

95 Lu H, Liu X M, Gong Y, et al. Optical bistability in MIM plasmonic Bragg waveguides with Kerr nonlinear defects. Appl Opt, 2011, 50: 1307-1311

96 Wang G X, Lu H, Liu X M. Trapping of surface plasmon waves in graded grating waveguide system. Appl Phys Lett, 2012, 101: 013111

97 Hau L V, Harris S E, Dutton Z, et al. Light speed reduction to 17 meters per second in an ultracold atomic gas. Nature, 1999, 397: 594-598

98 Bigelow M S, Lepeshkin N N, Boyd R W. Superluminal and slow light propagation in a room temperature solid. Science, 2003, 301: 200-202

99 Okawachi K, Biglow M S, Sharping J E, et al. Tunable all-optical delays via Brillouin slow light in an optical fiber. Phys Rev Lett, 2005, 94: 153902

100 Sandtke M, Kuipers L. Slow guided surface plasmons at telecom frequencies. Nat Photon, 2007, 1: 573-576

101 Hao R, Cassan E, Roux X L, et al. Improvement of delay-bandwidth product in photonic crystal slow-light waveguides. Opt Express, 2010, 18: $16309-16319$

102 Lu W T, Huang Y J, Casse B D F, et al. Storing light in active optical waveguides with single-negative materials. Appl Phys Lett, 2010, 96: 211112

103 Jang M S, Atwater H. Plasmonic rainbow trapping structures for light localization and spectrum splitting. Phys Rev Lett, 2011, 107: 207401

104 Stockman M I. Nanofocusing of optical energy in tapered plasmonic waveguides. Phys Rev Lett, 2004, 93: 137404

105 Wang G X, Lu H, Liu X M. Gain-assisted trapping of light in tapered plasmonic waveguide. Opt Lett, 2013, 38: 558-560

$106 \mathrm{Yu} \mathrm{Z}$, Veronis G, Fan S, et al. Gain-induced switching in metaldielectric-metal plasmonic waveguides. Appl Phys Lett, 2008, 92: 041117

Open Access This article is distributed under the terms of the Creative Commons Attribution License which permits any use, distribution, and reproduction in any medium, provided the original author(s) and source are credited. 